\title{
Preamble Design for Non-contiguous Spectrum Usage in Cognitive Radio Networks
}

\author{
Shulan Feng ${ }^{1}$, Heather Zheng ${ }^{2}$, Haiguang Wang ${ }^{1}$, Jinnan Liu ${ }^{1}$, Philipp Zhang ${ }^{1}$ \\ Hisilicon Technologies, Beijing, China ${ }^{1}$; Department of Computer Sciences, University of California, Santa Barbara, USA ${ }^{2}$ \\ \{fengsl, wanghaiguang, liujinnan,pzhang\}@huawei.com, htzheng@cs.ucsb.edu
}

\begin{abstract}
Cognitive radios can significantly improve spectrum efficiency by using locally available spectrum. The efficiency, however, depends heavily on their transceiver design. In particular, being able to use non-contiguously aligned spectrum bands simultaneously is a critical requirement. Prior work in this area requires a control channel so that transmitter/receiver pairs can synchronize on their spectrum usage patterns. However, this approach can suffer from high cost and control congestion. In this paper, we propose an in-band solution for informing receivers the spectrum usage patterns. By judiciously designing packet preambles, we embed the spectrum usage patterns in each data packet. Using the legacy 802.11 preamble structure, we focus on choosing the appropriate preamble sequences to maintain reliable packet detection in the presence of noise and interference. We verify our design using simulation and show that it can lead to reliable packet transmissions comparable to those of contiguous spectrum usage. We also identify the impact of interference on our design and propose refinements to choose the preamble sequence using information on the interference.
\end{abstract}

\section{INTRODUCTION}

Cognitive radios are the ideal solution to the artificial spectrum scarcity problem. Instead of using statically assigned spectrum, cognitive radios can operate on unused spectrum channels in their local neighborhoods without disrupting the operations of existing spectrum owners (primary users). Such opportunistic spectrum access not only provides available spectrum to new wireless technologies but also significantly improves spectrum utilization.

The amount of spectrum usable to cognitive radios depends on not only the primary user's activities but also the cognitive radio's transceiver design. Traditional radio transceivers, like those of $\mathrm{WiFi}$ radios, operate on contiguous spectrum frequencies. For example, a $5 \mathrm{MHz}$ transceiver can transmit only if it detects an idle contiguous $5 \mathrm{MHz}$ spectrum band. If the band is occupied by a narrowband user (e.g. a $200 \mathrm{KHz}$ wireless device), the transceiver treats the entire $5 \mathrm{MHz}$ band as busy. In this case, $96 \%$ of spectrum is wasted. To utilize spectrum efficiently, cognitive radios should be able to occupy spectrum bands that are non-contiguously aligned in frequency [1][2][9]. In this case, the $5 \mathrm{MHz}$ spectrum is divided into subbands, and cognitive radios can operate on multiple idle subbands simultaneously even if they are not contiguous in frequency.

Non-contiguous spectrum usage, however, faces many challenges[1]. In particular, because cognitive radios can configure spectrum usage flexibly, a key challenge is how to identify the pattern of spectrum usage at each receiver to decode the message successfully. Prior work[2][4][5] assumes that each transmitter informs its receiver the spectrum usage explicitly via a control channel. While feasible for infrastructure networks, this approach faces the following limitations. First, it requires a dedicated out-of-band control channel so that radios can exchange control information without interfering with primary users. The cost is rather high, especially for ad hoc networks. Second, it makes radio more complex, requiring either an extra control radio or tight synchronization among radios to enable time sharing among control and data transmissions. Finally, it also faces the problem of scalability, particularly under heavy load and as the number of subbands becomes large.

In this paper, we consider an alternative approach to identify spectrum usage patterns. We propose an in-band solution where information on spectrum usage patterns is embedded in data packets themselves. Specifically, the information is embedded in the preamble of each data packet, allowing the transmitter to adapt spectrum usage on a per-packet basis. Located at the beginning of each data packet, the preamble is a set of predefined sequences used for time synchronization and frequency and channel estimation. By judiciously designing preamble sequences to match spectrum usage patterns, our approach implicitly embeds the information in the preamble. Once the receiver successfully detects and decodes the preamble, it can use the spectrum pattern information to decode the data packet properly.

We illustrate our design using the legacy 802.11-2007 OFDM[3] system. The OFDM system is natural for noncontiguous spectrum transmissions since the subcarriers are modulated separately. The transmitter can configure (noncontiguous) spectrum usage by enabling a subset of the subcarriers. Because the preambles are transmitted using the same (and unknown) set of non-contiguous subcarriers as the data, the main challenge we face is how to design the preambles so that they can be reliably detected and decoded by the receiver in the presence of noise and interference.

Our key contributions and findings include:

- We propose an in-band solution for informing receivers the spectrum usage patterns. It differs from prior work by not relying on any control channel. To the best of our knowledge, we are the first to propose this solution.

- By judiciously designing packet preambles, we allow receivers to identify spectrum usage patterns on a perpacket basis. As such, transmitters can dynamically adapt the spectrum patterns to environment conditions and 
primary user activities.

- Our design starts from the legacy IEEE 802.11-2007 OFDM system and is backward compatible, allowing the cognitive radios to communicate with legacy devices.

- Using theoretical analysis and experiments, we examine the performance of proposed solution under different interference conditions. Our results show that the performance depends heavily on the percentage of the available spectrum and the signal to interference and noise ratio (SINR). In particular, under strong interference, non-contiguous spectrum usage faces performance degradations unless the receiver can filter out the interference.

- When the receiver can filter out the interference in the unavailable subcarriers, non-contiguous spectrum usage can achieve the same performance as contiguous spectrum transmission that uses the same total transmission power.

The rest of the paper is organized as follows. As background, we introduce in Section II the preamble design of the 802.11-2007 OFDM systems as well as the corresponding synchronization algorithm. In this case, the preamble design is targeted for contiguous spectrum usage. In Section III, we present the proposed preamble design in the context of noncontiguous spectrum usage. Using simulations, we examine the proposed solution under different network settings in Section IV. We discuss in Section V additional considerations and open problems. Finally, we conclude in Section VI.

\section{BACKGROUND: PREAMBLE IN 802.11-2007}

\section{A. Preamble structure}

As a part of each data packet, the preamble is a set of predefined sequences that are used for time synchronization, frequency estimation and channel estimation. In the 802.112007 OFDM system, the preamble consists of 10 identical Short Training Symbols (STS) and 2 identical Long Training Symbols (LTS) separated by a guard interval as shown in Figure 1. Short training symbols are used for packet detection, coarse frequency estimation and timing synchronization. Long training symbols are used for fine timing synchronization, fine frequency estimation and channel estimation.

Both STS and LTS are generated by periodically extending

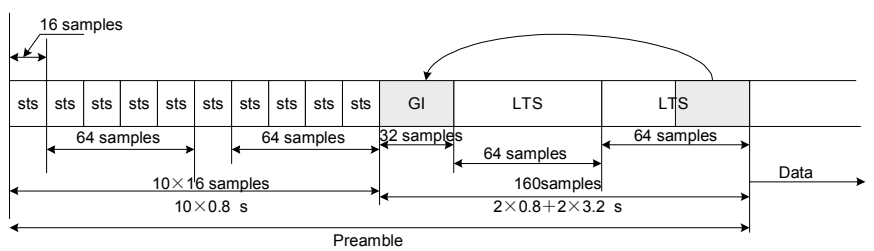

Fig.1: The preamble structure of the 802.11-2007 OFDM system
$x(n)=\sum_{k=-N_{S T} / 2}^{N_{S T} / 2} X_{k} e^{j \frac{2 \pi k n}{N_{F F T}}}$, for $\mathrm{n}=0,1, \ldots \mathrm{N}_{\mathrm{FFT}}-1$

where $\mathrm{N}_{\mathrm{FFT}}=64$ and $\mathrm{N}_{\mathrm{ST}}=52$. For STS, $X_{-26,26}=$ $S_{-26,26}=\sqrt{13 / 6}=\{0,0,1+\mathrm{j}, 0,0,0,-1-\mathrm{j}, 0,0,0,1+\mathrm{j}, 0,0,0,-$ $1-\mathrm{j}, 0,0,0,-1-\mathrm{j}, 0,0,0,1+\mathrm{j}, 0,0,0,0,0,0,0,-1-\mathrm{j}, 0,0,0,1-$ $\mathrm{j}, 0,0,0,1+\mathrm{j}, 0,0,0,1+\mathrm{j}, 0,0,0,1+\mathrm{j}, 0,0,0,1+\mathrm{j}, 0,0\}$. The factor of $\sqrt{13 / 6}$ is used to normalize the average power of the resulting OFDM symbol, which utilizes 12 out of 52 subcarriers. For LTS, $X_{-26,26}=L_{-26,26}=\{1,1,-1,-1,1,1,-1$, $1,-1,1,1,1,1,1,1,-1,-1,1,1,-1,1,-1,1,1,1,1,0,1,-1,-$ $1,1,1,-1,1,-1,1,-1,-1,-1,-1,-1,1,1,-1,-1,1,-1,1,-1$, $1,1,1,1\}$

\section{B. Packet detection, coarse frequency estimation and timing synchronization}

STSs are used for packet detection, coarse frequency estimation and timing synchronization. To illustrate the difference between contiguous and non-contiguous spectrum usage, we use a set of widely used mechanisms for these tasks[10]. The synchronization procedure of 802.11 receivers is shown in Fig. 2.

For packet detection and coarse timing estimation, we use the delay-correlation algorithm defined by the following equation:

$$
C(k)=\left|\sum_{m=0}^{M-2} \frac{\sum_{n=0}^{N_{S T S}-1} r\left(k+m \times N_{S T S}+n\right) \times r^{*}\left(k+m \times N_{S T S}+n+N_{S T S}\right)}{\sum_{n=0}^{N_{S T S}-1}\left|r\left(k+m \times N_{S T S}+n+N_{S T S}\right)\right|^{2}}\right|^{2}
$$

where $r(k)$ denotes the discrete complex time domain received baseband signal. $\mathrm{N}_{\mathrm{STS}}=16$ is the length of the repetition sequences of the STSs and $\mathrm{M}=10$ is number of repetition sequence of the STSs. A packet is assumed to be detected if $C(k)$ is greater than a given threshold for a specific $k_{P D}$. The coarse timing on the beginning of a packet can be derived by searching the $\widetilde{k}_{F S}=k$ that maximizes $C(k)$ within $\left[k_{P D}, k_{P D}+W_{c t}-1\right]$, where $W_{c t}$ is the length of the coarse timing search window. Note that in this paper, if the coarse timing deviates by more than half of STS, or, 8 samples, the packet is treated as being falsely detected.

Next, the coarse frequency offset is estimated as:

$$
\begin{aligned}
& \Delta f_{C F E}=\frac{1}{2 \pi \times T_{\text {sample }} N_{S T S}} \\
& \arg \left\{\sum_{m=0}^{M-2 N_{S T S}-1} r\left(\tilde{k}_{F S}+m \times N_{S T S}+n\right) \times r^{*}\left(\tilde{k}_{F S}+m \times N_{S T S}+n+N_{S T S}\right)\right\}
\end{aligned}
$$

\section{Fine timing and frequency estimation}

The LTSs are used for fine timing and frequency estimation. The following equation is used to derive the fine frequency estimation: 
$\Delta f_{F F E}=\frac{1}{2 \pi \times T_{\text {sample }} N_{L T S}}$

$\arg \left\{\sum_{n=0}^{N_{L T S}-1} r^{\prime}\left(\widetilde{k}_{F S}+160+24+n\right) \times\left\{r^{\prime}\left(\tilde{k}_{F S}+160+24+n+N_{L T S}\right)\right\}^{*}\right\}$

where $\mathrm{N}_{\mathrm{LTS}}=64 . \widetilde{k}_{F S}$ is the coarse timing estimation for the beginning of packet. $\left(\tilde{k}_{F S}+160\right)$ is the coarse timing estimation for the beginning of LTS. Additional 24 samples are moved to avoid the multipath delay and coarse timing deviation. $r^{\prime}(\cdot)$ is the output of $r(\cdot)$ corrected by the coarse frequency estimation.

To derive the fine timing estimation, we use the crosscorrelation between the local sequence and the receive sequence within a search window. The fine timing estimation is derived by searching for the $\tilde{k}_{F S}=k$ that maximizes $P(k)$ in the window $\left[\widetilde{k}_{F S}+160-8, \widetilde{k}_{F S}+320+8\right]$. In this case,

$$
P(k)=\sum_{n=0}^{159} r^{\prime \prime}(k+n) \times p^{*}(n)
$$

where $p(n), n=0,1, \ldots 159$ is the local long training sequence in the time domain. $r^{\prime \prime}(\cdot)$ is the output of $r^{\prime}(\cdot)$ corrected by the fine frequency estimation.

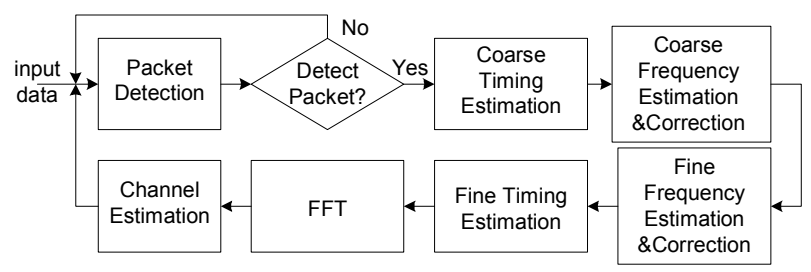

Fig. 2: The synchronization procedure of the 802.11-2007 OFDM receiver

\section{PreAmble Design For Non-CONTIGUOUS SPECTRUM USAGE}

In Section II, we briefly overview the existing preamble design for contiguous spectrum usage. We now describe our proposed preamble design for non-contiguous spectrum usage. Note that we apply a similar preamble structure to achieve the backward compatibility, but judiciously design STS and LTS sequences to embed information of spectrum usage patterns and enable reliable detection and decoding.

The proposed preamble consists of 10 identical STSs and 2 identical LTSs, separated by a guard interval.

- The short training symbol is generated by a sequence with 12 elements which are modulated independently to subcarrier -24,-20,-16,-12,-8,-4, 4, 8, 12, 16, 20, 24. If any of these subcarriers are unavailable, they are modulated by zero. The rest of the subcarriers are modulated by zero.

- The long training symbol is generated by a sequence with 52 elements which are modulated independently to subcarrier -26 -1 and 1 26. Subcarrier 0 and unavailable subcarriers in non-contiguous spectrum mode are modulated by zero.

We now describe the details of our design on short training sequences and long training sequences. Note that all these sequences are transmitted to the receiver using the same set of (available) non-contiguous subcarriers as the data.

\section{A. Design of short training sequence}

As discussed before, STSs are used for packet detection, coarse frequency offset estimation and time synchronization. Since the length of short sequence in time domain is short (16), we choose not to embed the spectrum patterns in STS. Hence, the challenge of our design is how to perform the packet detection and synchronization when the STSs are transmitted using a set of subcarriers unknown to the receiver.

As shown in Section II, the performance of packet detection algorithm depends on the delay correlation and is independent of the correlation property of the STS sequence. So the main design requirement for STS is to minimize the Peak-to-Average Power Ratio (PARR).

In terms of the sequence, we consider the following widelyused designs:

- $\quad$ STS -- the legacy 802.11 short training sequences.

- $\quad$ FZC -- the Frank-Zadoff-Chu sequence[7]. The element $a_{k}$ in the sequence is defined by $a_{k}=W_{12}{ }^{k^{2} / 2}, k=0,1, \ldots, 11$, $W_{12}=e^{-j 2 \pi r / 12}, r=5$.

- $\quad$ SR -- the Shapiro-Rundin sequence[8].

- Newman-- the Newman sequence[8], a special type of the FZC sequence with $r=1$.

- GCL-- the generalized chirp-like poly-phase sequence[7] which is generated from the FZC sequence with $r=1$.

Figure 3 compares these commonly used sequences in terms of their average PAPR under different available spectrum ratios. The available spectrum ratio is defined as $N_{\text {avail }} / N_{\text {total }}$, where $N_{\text {total }}$ is the total number of subcarriers $\left(N_{\text {total }}\right.$ $=52$ for the 802.11 OFDM system), and $N_{\text {avail }}$ is the number of the available subcarriers. Results of Figure 3 show that the legacy 802.11 short training sequences lead to the lowest PAPR in most cases. Therefore, we choose this sequence as our STS. Note that we also examine the impact of the patterns of available subcarriers, such as randomly distributed across $N_{\text {total }}$ subcarriers or clustered. The same conclusions are drawn.

\section{B. Design of long training sequence}

Long training symbols are used for channel estimation, fine timing and spectrum usage pattern identification in noncontiguous spectrum transmission mode. The requirements for the long training sequence are:

- Good periodic auto-correlation to provide good timing synchronization.

- Good cross-correlation property to reliably identify spectrum usage patterns.

- Large family size to support large number of non- 
contiguous spectrum usage patterns.

\section{- Low PAPR.}

Both the Gold sequence and the FZC sequence are good candidates because of their auto-correlation and crosscorrelation properties[6][7]. Each Gold sequence is of length $2^{n}-1$, and if $n=6$, is of length 63 . When $n=6$, It is easy to show that there exist 65 Gold sequences with $M_{p} / M_{\max }=63 / 17=3.7$, where $M_{p}$ is peak magnitude of periodic autocorrelation, $\mathrm{M}_{\max }=\max \left(\mathrm{M}_{\mathrm{a}}, \mathrm{M}_{\mathrm{c}}\right), \mathrm{M}_{\mathrm{a}}$ is peak out-of-phase autocorrelation magnitude, $M_{c}$ is peak cross correlation magnitude[6]. Because the long training sequences are modulated across 52 subcarriers, we truncate the gold sequence to 52 which leads to $\mathrm{M}_{\mathrm{p}} / \mathrm{M}_{\max }=52 / 30=1.7$.

For the FZC sequence, the out-of-phase autocorrelation magnitude is 0 . The cross correlation magnitude between any two FZC sequences of odd length $\mathrm{N}$, obtained from the two different primitive $\mathrm{N}^{\text {th }}$ roots of unity $e^{j 2 \pi v / N}$ and $e^{j 2 \pi u / N}$, is constant and equal to $\sqrt{N}$, if $(u-v)$ is relatively prime to $\mathrm{N}[7]$. We set $\mathrm{N}=53$, producing 52 sequences with $\mathrm{M}_{\mathrm{p}} / \mathrm{M}_{\max }=53 / \sqrt{53}=7.280$. Because only 52 subcarriers can be used to transmit the long training sequence, we replace the $27^{\text {th }}$ element of FZC with $\mathrm{N}=53$ to 0 and modulate it to subcarrier 0 . This makes the $\mathrm{M}_{\mathrm{p}} / \mathrm{M}_{\max }=52 / 9.2553=5.6184$.

From the above analysis, we can see that the correlation property of FZC sequences is better than the Gold sequence under similar family sizes. To verify our analysis, in Figure 4 we use simulations to compare the correlation property of these sequences under different available spectrum allocation ratios. For every bar in the Figure, the upper point is the maximum value in the 1000 Monte-Carlo simulations and the lower point is the minimum, and the middle point is the mean. These results confirm that the FZC sequence provides a better correlation property than the Gold sequence. Therefore, our design selects a family of the FSC sequence with $\mathrm{N}=53$ as the long training sequences.

Finally, we also found that the PAPR of all FZC sequences in the selected FZC sequence family are less than $7.5 \mathrm{~dB}$ as then available subcarrier ratios ranging from $40 \%$ to $100 \%$.

\section{Map the FZC sequence to spectrum usage pattern}

The ultimate goal of dynamic spectrum usage is to configure the 52 subcarriers, which means $\left(2^{52}-1\right)$ spectrum usage patterns. It is, however, impractical to identify the sequence from such a large $\left(2^{52}-1\right)$ sequence set. To reduce the set of sequences, we propose to make reasonable assumptions on the primary user's activities. For example, if at any time, there is at most one narrow band that is unavailable among the 52 subcarriers, we can simplify (and limit) the pool of the spectrum usage patterns. One solution is to map the start position of the unavailable subcarrier to one sequence, which requires only 52 sequences. Another solution is to divide the total 52 subcarriers to multiple regions, e.g. 5 regions, and restrict the usage on the region level. This leads to $\left(2^{5}-1\right)$ sequences.

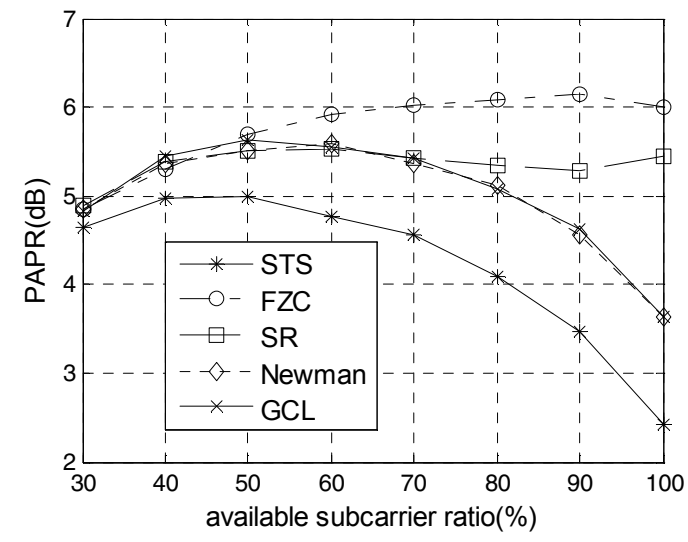

Fig. 3: The PAPR of different short training sequences under different available subcarriers ratios averaged over 1000 simulation runs.

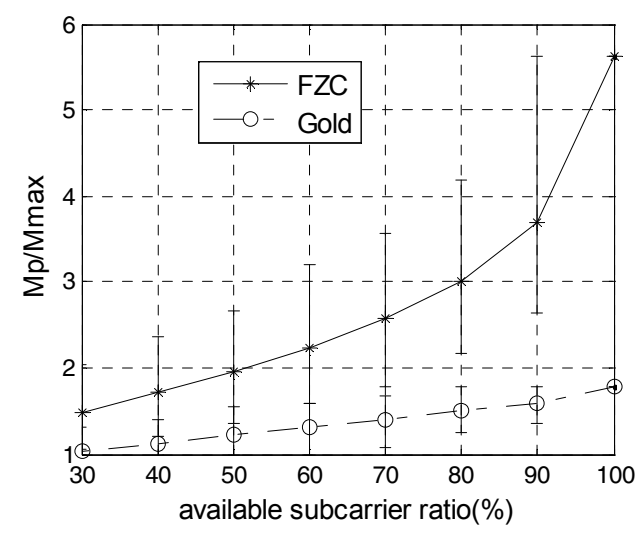

Fig. 4: The correlation properties of the gold sequences and FZC sequences under different available spectrum

Instead of making strict assumptions on primary users and spectrum availability, we propose a general approach that scales with the number of available channels. Specifically, we divide the total spectrum to $M$ regions. The number of spectrum patterns that contain at most $\mathrm{P}$ narrowband primary users in different parts $Q$ is $Q(M, P)=\left(1+C_{M}{ }^{1}+C_{M}{ }^{2}+\ldots+C_{M}{ }^{P}\right)$. We can select $P$ first according to the knowledge of primary users and prior spectrum statistics, and then select the maximum $\mathrm{M}$ such as $\mathrm{Q}(\mathrm{M}, \mathrm{P})$ is no more than the size of correlation sequences. For example, if there are at most two narrow band primary users in different subbands, we can select $\mathrm{P}=2$ and $\mathrm{M}=9$ which produces $\mathrm{Q}(9,2)=46<52$.

\section{PERFORMANCE EVALUATION}

In this section, we evaluate the performance of the proposed preamble design for non-contiguous spectrum usage. Note that in our design, the preamble is not only used for frequency estimation, timing estimation, channel estimation, but also for spectrum usage pattern identification. Among all these requirements, the synchronization procedure in the noncontiguous usage is similar to those of the contiguous spectrum usages except that our solution chooses one among multiple local sequences that provides the maximum correlation as the transmission sequence. 


\section{A. Packet detection, coarse timing and coarse frequency estimation}

Figure 5 shows the packet detection probability in an isolated scenario without any interference. We examine the performance under different available spectrum ratios. We observe that with no interference, the packet detection probability is independent of the spectrum usage patterns. This is because the transmission power is normalized in time domain. As we have stated in Section II, every modulated subcarrier's signal is enhanced by a factor of $\sqrt{13 / 6}$ to normalize the average power of the resulting OFDM symbol in contiguous spectrum transmission mode, and by a factor of $\sqrt{52 / N_{\text {STS-non }}}$ in non-contiguous mode, where $\mathrm{N}_{\text {STS-non }}$ is the number of active subcarriers. Even under different available spectrum ratios, the average SNR in time domain is the same as that of the contiguous spectrum usage.

Figure 6 shows the average packet detection probability under different interference levels and different available spectrum ratios when SNR is $7 \mathrm{~dB}$ over 1000 Monte-Carlo simulations. We observe that the packet detection probability deteriorates with the level of interference. This is because the packet detection probability depends on the SINR:

$$
\operatorname{SINR}=\frac{P_{\text {signal }}}{P_{\text {interference }}+P_{\text {noise }}}=\frac{1}{P_{\text {interference }} / P_{\text {signal }}+P_{\text {noise }} / P_{\text {signal }}}
$$

where $P_{\text {interference }}=($ unavailable spectrum ratio $) \times($ interference to signal ratio) $\times P_{\text {signal }}$ In Figure $6, P_{\text {noise }}$ and $P_{\text {signal }}$ remain the same in different cases, so the performance depends only on the available spectrum ratio and the interference to signal ratio. For example, the false detection probability for $70 \%$ available spectrum ratio and $0 \mathrm{~dB}$ interference to signal ratio is same for the $85 \%$ available spectrum ratio and $0 \mathrm{~dB}$ interference to signal ratio.

The relationship between the available spectrum ratio and the interference power can be used by the transmitter to determine the transmission band. For example, if the system intends to limit the false detection probability to below $10 \%$, then when $\mathrm{SNR}=7 \mathrm{~dB}$, the transmitter will not transmit if the available spectrum is lower than $85 \%$ and the interference to signal ratio is greater than $3 \mathrm{~dB}$.

Figure 7 shows the variance of coarse frequency estimation in different interference levels and different available spectrum ratios when the SNR is $7 \mathrm{~dB}$ and the frequency offset is $200 \mathrm{KHz}$. We get similar conclusions because the performance of frequency estimation depends on the SINR, and thus the unavailable spectrum ratio and interference to signal ratio. For example, the coarse frequency estimation variance for $80 \%$ available spectrum and $3 \mathrm{~dB}$ interference to signal ratio is similar to that of $90 \%$ available spectrum ratio and $6 \mathrm{~dB}$ interference to signal ratio.

\section{B. Fine timing and frequency estimation and spectrum usage pattern identification}

The performance of fine frequency timing, fine frequency estimation and spectrum usage pattern identification also depends heavily on the SINR. As in Section IV-A, the

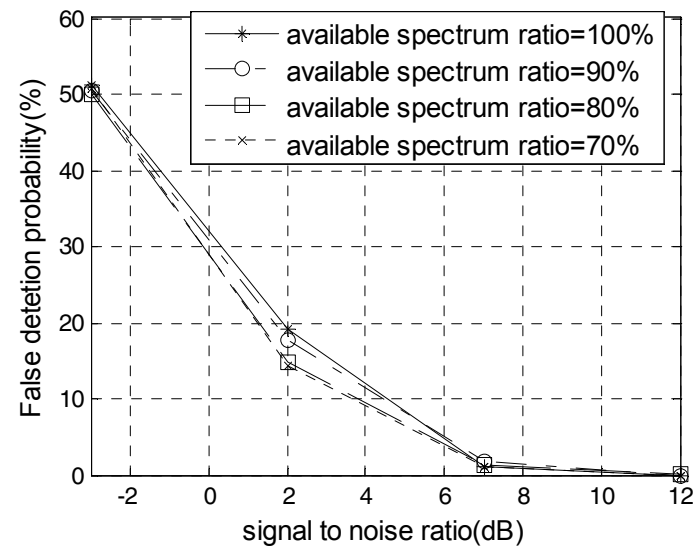

Fig. 5: The packet detection probability under different available spectrum ratios without interference

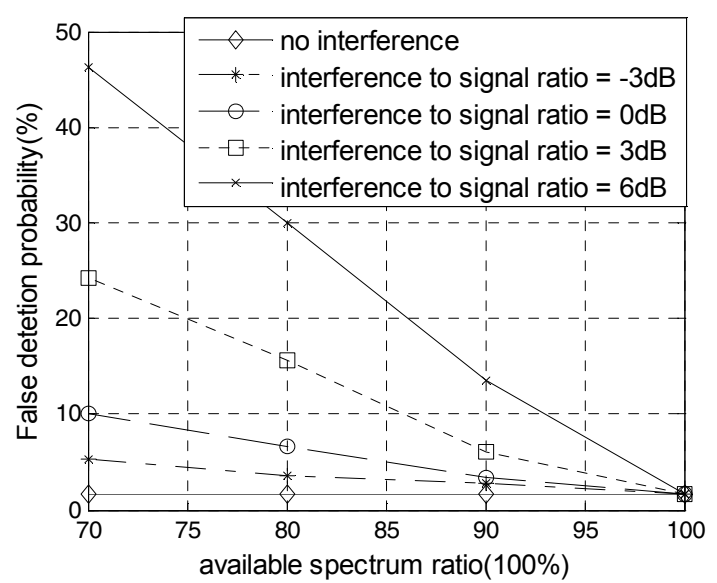

Fig. 6: The packet detection probability under different interference levels and different available spectrum ratios when $\mathrm{SNR}=7 \mathrm{~dB}$

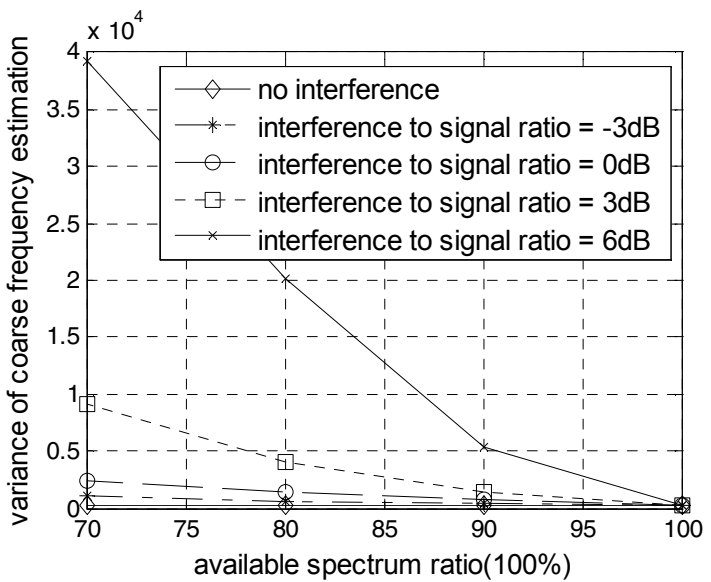

Fig. 7: The variance of coarse frequency estimation under different interference levels and different available spectrum ratios when $\mathrm{SNR}=7 \mathrm{~dB}$ 
performance of fine timing, frequency estimation and spectrum usage pattern identification depends heavily on the SINR. However, since fine estimation uses LTSs with more elements than those of STSs, the synchronization performance becomes much robust. For example when $\mathrm{SNR}=7 \mathrm{~dB}$, there is no false spectrum usage pattern identification for $60 \%$ available spectrum allocation ratio when the interference to signal power is less than $9 \mathrm{~dB}$, and for $80 \%$ available ratio when the interference to signal power is less than $18 \mathrm{~dB}$. Such performance advantage motivates us to "upgrade" the preamble design to use four identical long training symbols. The disadvantage, however, is that the transmission becomes no longer backward compatible to the legacy 802.11 OFDM system.

\section{DISCUSSION AND RELATED WORK}

In this paper, we present a preamble design for noncontiguous spectrum usage, which embeds spectrum usage patterns in data packets. We use the 802.11-2007 OFDM preamble as an example to demonstrate our ideas and to examine the performance of the proposed design in contiguous and non-contiguous spectrum usages. Our design can easily be extended to other systems, including the $3 \mathrm{G}$ long term evolution systems which coexist with narrowband GSM users, or the WRAN systems defined in IEEE 802.22 which coexist with narrowband Part 74 users. Preamble design for these systems will be a future work.

Another requirement of our design is backward compatibility, which can limit the choice of sequences. As discussed in Section IV, we can consider alternative structures such as using 4 identical long training symbols as preamble. However, it requires a very different synchronization procedure, such as applying a two-step frequency offset estimation (one for fractional frequency estimation and another for integral frequency estimation). To take advantage of this robust structure, many function blocks of the legacy 802.11 devices must be modified. We plan to look into this issue in the future work.

Prior work[5][7] evaluates the synchronization performance in non-contiguous spectrum usage, assuming the spectrum pattern is known at the receiver by some means, for example, by signaling using a common channel. Our work differs from them by considering the detection of noncontiguous spectrum usage patterns, and providing explicit analysis on the interaction between the available spectrum ratios and the interference power. The work in [5] also evaluated the synchronization in different interference levels and different available spectrum ratios, but did not identify the interaction between the two factors and their impact on packet detection. Finally, the preamble proposed in [5] is not backward compatible to the legacy of 802.11 . The analysis and simulations in [7] do not consider the interference. Therefore it is an ideal case assuming the receiver knows the non-contiguous spectrum usage patterns and can filter out all the interference. Further, this approach normalizes transmit power per subcarrier, so that the performance of synchronization decreases when using non-contiguous spectrum. In our work, we normalize the average transmit power per symbol, so the synchronization performance is same in the contiguous and non-contiguous spectrum if the interference is filtered out.

\section{CONCLUSTION}

In this paper, we propose an implicit in-band signaling mechanism to enable non-contiguous spectrum usage. We design the preamble sequence based on the IEEE 802.11-2007 OFDM system, so that the transmitter can inform receiver its spectrum usage on a per-packet basis. Using both theoretical analysis and simulation experiments, we verify our design concept and examine the performance under different configurations. We show that the performance depends heavily on the available spectrum ratio and the interference to signal power ratio. This information, if available to the transmitter, allows the transmitter to choose the spectrum usage patterns intelligently and improve spectrum usage efficiency. Compared to existing solutions, our solution differs significantly because it does not require any dedicated control channel. To the best of our knowledge, our work is the first to address non-contiguous spectrum usage pattern without requiring any control channel.

\section{REFERENCES}

[1] T.A. Weiss, F.K. Jondral, Spectrum Pooling: An Innovative Strategy for the Enhancement of Spectrum Efficiency, IEEE Radio Comminucation, pp. S8- S14, March 2004.

[2] M. Krondorf, T.J. Liang and G. Fettweis, On Synchronization of Opportunistic Radio OFDM Systems, Proc. of IEEE VTC Spring 2008, May 2008.

[3] 802.11-2007, IEEE Standard for Information technologyTelecommunications and metropolitan area networks - Specific requirements - Part 11: Wirelss LAN Medium Access Control and Physical Layer Specifications, June 12, 2007.

[4] A. M. Wyglinski, Effects of Bit Allocation on Non-contiguous Multicarrier-based Cognitive Radio Transceivers, Proc. of IEEE VTC Fall 2006, September 2006.

[5] T. Weiss, A. Krohn, and F. Jondral, Synchronization Algorithms and Preamble Concepts in Spectrum Pooling Systems, Proc. of IST Mobile and Wireless Telecommunications Summit, 2003.

[6] D.Sarwate, M.Pursley, Crosscorrelation Properties of Pseudorandom and Related Sequences, Proc. of the IEEE, Vol.68, pp.593-619, May 1980.

[7] B. M. Popovic, Generalized Chirp-like Polyphase Sequences with Optimum Correlation Properties, IEEE Transactions on Information Theory, vol. 38, no. 4, pp 1406-1409, July 1992.

[8] S. Boyd, Multitone Signals with Low Crest Factor, IEEE Transactions on Circuits and Systems, CAS-33(10):1018-1022, October 1986.

[9] I. F. Akyildiz, W.-Y. Lee, M. C. Vuran, and S. Mohanty, NeXt Generation/Dynamic Spectrum Access/Cognitive Radio Wireless Networks: A Survey. Computer Networks Journal (Elsevier), 2006.

[10] F. Wu, M. A. Abu-Rgheff, Time and Frequency Synchronization Techniques for OFDM Systems operating in Gaussian and Fading Channels: A Tutorial, Proc. of the 8th Annual Postgraduate Symposium on The Convergence of Telecommunications,Networking and Broadcasting (PGNET), June 2007. 